\title{
Successfully translating conservation research into practice and policy: concluding thoughts
}

\author{
NATHALIE PETTORELLI \\ Zoological Society of London \\ PETER N. M. BROTHERTON \\ Natural England \\ ZOE G. DAVIES \\ Durrell Institute of Conservation and Ecology (DICE) \\ NANCY OCKENDON, WILLIAM J. SUTHERLAND \\ University of Cambridge \\ and \\ JULIET A. VICKERY \\ RSPB Centre for Conservation Science
}

In the Anthropocene, when our environment is changing rapidly and the windows of opportunity for action to prevent further biodiversity loss are narrow, conservation researchers are increasingly encouraged to think and operate beyond the traditional approaches of producing peer-reviewed papers and presenting results to other members of the research community. Indeed, the perception that researchers belong in their ivory tower, from which they deliver evidence for others to interpret, disseminate and use in decisionmaking, is thankfully now widely recognised as outdated. The rise of fake news, a deliberate lack of consideration for scientific evidence, and changes to the ways of assessing the value of researchers' work probably all play a role in supporting this shift in perception. Moreover, for many researchers, the prospect of their work 'making a difference' and having an impact on wider society is at least as great a motivation for doing research as generating new knowledge, however interesting that may be. In addition, researchers and research institutions are nowadays not only required to contribute to advancing knowledge, but also play a part in societal development. Impact thus matters to a growing number of researchers and funders, and it increasingly shapes the functioning of research institutions worldwide. 
Research impact can come in a plethora of forms, but the pathway to delivery will typically involve negotiating the interface between research and policy/practice successfully. One recurring theme that emerges in this book is the need for close working relationships between those generating evidence and the practitioners and policy-makers that apply it. The nature, quality and regularity of these interactions are instrumental in ensuring that pertinent evidence underpins solid decision-making. For too long, one of the biggest misconceptions about the interface between research and policy/practice has been that it follows a linear model, whereby decision-makers pose questions, and researchers generate appropriate evidence, which is then used by the decision-makers to make well-informed choices. We hope that this book helps to further dispel this myth.

Instead, we lay out some of the potentially more complex models by which researchers, policy-makers and practitioners can be brought together, across all stages of the knowledge generation, exchange and application process. These models range from collaborative efforts to identify future research priorities through to co-producing projects that can provide outputs that address clear and topical policy or practice needs. Within a policy arena, such relationships require time to establish, and this can be challenging when political leaders change position relatively frequently. Researchers therefore need to consider being 'in it for the long haul', possibly well after the papers have been published and the novelty factor has worn off. Moreover, balancing the sometimes slow pace of change in policy with a research world always looking for new and exciting opportunities can be challenging. Close collaboration with policy staff within non-governmental organisations can be one way to overcome this problem, as they are often well networked within the decision-making communities and have a good understanding of how the 'system' works. Equally, developing meaningful relationships with those holding non-political appointments in government (such as in government agencies or the civil service) can prove fruitful. Ultimately, while the methods adopted, for example to synthesise and present different types of information or assess the cost-effectiveness of a range of policy options, are fundamental steps to enable the use of research in decision-making, building mutual trust and respect between individual researchers and decision-makers can make the difference to whether the available evidence is used.

Another key relationship is that between scientists and the practitioners or project managers making conservation decisions on the ground. Building relationships with, and learning from, practitioners can provide a unique opportunity to gain detailed insights into how research supports (or fails to support) management interventions. Much work has been done to better connect policy-makers and researchers in various countries, but we are yet to provide similar national platforms for researchers to better connect with 
practitioners. This step matters, as the consequences of not using scientific evidence when making decisions about conservation interventions can be damaging, in terms of both wasting limited human and financial resources and failing to meet biodiversity objectives.

An important point we hope to have conveyed with this contribution is that societal change, and thus impact, can in some cases be secured without direct engagement with policy-makers and practitioners. The internet and social media have considerably changed the modus and speed with which researchers can communicate with the public, in effect making campaigning an accessible tool for everyone. However, such a strategy can come at a cost, and lead to unintended consequences. Importantly, the choices we make as individuals (such as avoiding products with palm oil or buying organic food) are often inseparable from the enabling environments (including social norms, the political and economic situation, and incentives to promote certain behaviours) in which we make them. Because of this, the most effective route to change often means targeting both the individual (for example by working with approaches that help change motivations, habits, emotional engagement and awareness) and the enabling environment.

While encouraging the improved use of evidence by practitioners and policy-makers, it is important to remain mindful of the intricacies of the multitude of factors that influence decision-making in both these domains. These may include layers of advice from colleagues and personal experience, as well as a myriad of multi-faceted social, economic and cultural factors. This can be a frustration to researchers, and may influence the nature and content of their communications with decision-makers and the type of relationships that are built. This deeply human dimension to working at the research-policy/practice interface remains underappreciated by many in conservation. Researchers that are inexperienced with the researchpolicy/practice interface may arguably achieve more by collaborating with communication or behavioural change experts from the initial stages of project through to completion, rather than by going it alone or only considering communication as an add-on extra once the results of a project are complete.

This book would have not seen the light of day if researchers around the world were not increasingly recognising the need to engage and collaborate with decision-makers from the outset, to ensure the value and timeliness of their work for conservation policy and practice. Our aim here was to ask a diversity of experts to respond to a single question, namely how best to ensure impact is realised. Most of our contributors agree on one thing: researchers need to use a variety of approaches and invest in a range of different relationships to make sure high-quality evidence is co-developed and co-produced with relevant stakeholders. 
Some also point to the importance of providing increased formal or informal training to current and future conservation researchers in the skills that are needed to work productively at the research-policy/practice interface. Teaching the next generation about effective knowledge exchange and how to interact with governmental and parliamentary procedures within the confined walls of universities can only go so far. Early-career researchers will likely model their behaviour and approaches on the senior researchers that they are exposed to, and learning in a classroom setting will never replace firsthand experience. Moreover, while establishing mutual trust and understanding between researchers and decision-makers is vital, it may be challenging to find the space to meet and develop relationships when both communities are subject to different work priorities, constraints and cultures. To address these issues, a number of initiatives have surfaced to increase and enhance opportunities for direct interactions between, and in-situ training for, researchers and decision-makers at all levels of seniority, many supported by research councils and learned societies.

Ultimately, our compilation of case studies and opinion pieces clearly demonstrates how engagement with policy and practice ultimately challenges us as researchers to individually confront our fears and impostor syndrome relating to our ability to generate good, useful and 'as certain as it gets' knowledge that may be appreciated and valued by society as a whole. It also challenges our egos, forcing us to realise that scientific evidence is, at the end of the day, only one of the many considerations shaping decisions. Finally, these case studies highlight how the research-policy/practice interface ultimately consists of a collection of individual research-policy/practice interfaces, shaped over the years by those who appreciate how rewarding collaborating together can be, but also understand that it requires long-term commitment. Within this book, we have tried to bring together collective wisdom of how each of us can best build our own interface, with the aim of equipping current and future generations of conservation researchers with the tools and knowledge to help them to decide how to best navigate the specific policy/practice context within which they work. We hope that this broad diversity of experience and advice will provide a valuable resource, enabling people interested in translating their research to bring about real-world change for the benefit of biodiversity. 